\title{
Modeling of cavities using the analytic modal method and an open geometry formalism
}

de Lasson, Jakob Rosenkrantz; Christensen, Thomas; Mørk, Jesper; Gregersen, Niels

Published in:

Optical Society of America. Journal A: Optics, Image Science, and Vision

Link to article, DOI:

10.1364/JOSAA.29.001237

Publication date:

2012

Document Version

Publisher's PDF, also known as Version of record

Link back to DTU Orbit

Citation $(A P A)$ :

de Lasson, J. R., Christensen, T., Mørk, J., \& Gregersen, N. (2012). Modeling of cavities using the analytic modal method and an open geometry formalism. Optical Society of America. Journal A: Optics, Image Science, and Vision, 27(7), 1237-1246. https://doi.org/10.1364/JOSAA.29.001237

\section{General rights}

Copyright and moral rights for the publications made accessible in the public portal are retained by the authors and/or other copyright owners and it is a condition of accessing publications that users recognise and abide by the legal requirements associated with these rights.

- Users may download and print one copy of any publication from the public portal for the purpose of private study or research.

- You may not further distribute the material or use it for any profit-making activity or commercial gain

- You may freely distribute the URL identifying the publication in the public portal 


\title{
Modeling of cavities using the analytic modal method and an open geometry formalism
}

\author{
Jakob Rosenkrantz de Lasson, ${ }^{\dagger}$ Thomas Christensen, ${ }^{\dagger}$ Jesper Mørk, and Niels Gregersen* \\ DTU Fotonik, Department of Photonics Engineering, Technical University of Denmark, Ørsteds Plads, \\ Building 343, DK-2800 Kongens Lyngby, Denmark \\ ${ }^{*}$ Corresponding author: ngre@fotonik.dtu.dk
}

Received September 27, 2011; revised March 12, 2012; accepted March 12, 2012; posted March 15, 2012 (Doc. ID 155363); published June 6, 2012

\begin{abstract}
We present an eigenmode expansion technique for calculating the properties of a dipole emitter inside a micropillar. We consider a solution domain of infinite extent, implying no outer boundary conditions for the electric field, and expand the field on analytic eigenmodes. In contrast to finite-sized simulation domains, this avoids the issue of parasitic reflections from artificial boundaries. We compute the Purcell factor in a two-dimensional micropillar and explore two discretization techniques for the continuous radiation modes. Specifically, an equidistant and a nonequidistant discretization are employed, and while both converge, only the nonequidistant discretization exhibits uniform convergence. These results demonstrate that the method leads to more accurate results than existing simulation techniques and constitutes a promising basis for further work. @ 2012 Optical Society of America
\end{abstract}

OCIS codes: $\quad 000.3860,050.1755,230.5750,230.7370,290.0290$.

\section{INTRODUCTION}

Quantum emitters embedded in optical microcavities, such as photonic crystals and micropillars, constitute an important platform for exploring a range of interesting physical phenomena as well as realizing quantum information devices. This includes a broad range of interesting features, including enhanced light-matter interactions, quantum entanglement, and single-photon emission [1]. The latter is intimately related to the Purcell effect that describes the enhancement or inhibition of the spontaneous emission rate (SER) of an emitter when positioned inside an optical cavity [2]. Enhancement of the SER is vital in the development of efficient and reliable single-photon sources in the scope of quantum information technology [3].

To obtain the desired functionality in such devices, accurate numerical modeling of the electromagnetic field is crucial. Numerical methods based on spatial discretization such as finite-difference time-domain (FDTD) [4] and the finite element method (FEM) []ㅡ ] are popular; however, the necessity of discretizing the entire computational domain leads to huge memory requirements for realistic device geometries. On the other hand, modal methods such as the Fourier modal method [6] and eigenmode expansion technique (EET) [7] are less memory demanding, and in addition the approaches themselves provide a better insight into governing physical mechanisms of interest. In this article, we formulate and demonstrate the application of the EET to a geometry without outer boundary conditions, the so-called open geometry that is commonly encountered in optics.

In modal methods, or rigorous coupled-wave analysis, the electromagnetic fields are expanded on a complete and orthonormal set of basis functions. The set of basis functions can be chosen as the set of eigenmodes supported by the optical environment under consideration, giving rise to the EET. These eigenmodes can be determined using Fourier analysis or by direct analytic determination of the eigenmodes. In a homogeneous medium, the eigenmodes are indeed plane waves, but in more advanced structures, such as the micropillar to be considered in this article, the complete set of eigenmodes includes a finite number of guided modes and a continuum of radiation modes.

A common issue for most of the suggested simulation techniques is that practical implementation enforces a finite-sized solution domain. In its simplest form, this implies the constraint that the field must vanish at the boundaries of the solution domain, which inevitably produces parasitic reflections at these metal-like boundaries [8] . As a means to reduce these effects, absorbing boundaries, the so-called perfectly matched layers (PMLs), were introduced [9]. The use of analytic eigenmodes in combination with PML was investigated by Bienstman and Baets [7,8], and numerically stable results using this technique were demonstrated [10]. However, the use of PMLs requires a set of parameters to be determined that define the boundary region, and convergence of the electric field upon adjustment of these parameters, toward that of an open geometry, is not guaranteed [11]. Thus, even PML does not fully eliminate the parasitic perturbations of the fields [12], and this inherent deficiency of finite-sized simulation domains motivates the introduction of an open geometry of infinite extent.

The open geometry has been treated by expansion of the eigenmodes on a Fourier-Bessel basis [13]. On the other hand, expansions on analytical eigenmodes are applied in [14], where an open geometry with radiation from a waveguide into free space is treated. There, an integral equation for the field at the interface between the waveguide and free space is derived and solved by a perturbative approach. First- and second-order solutions are presented, but the approximate solution procedure in practice limits the index contrasts that 
can be treated. To address this issue, an accelerated iteration procedure has been proposed [15].

In the present work, we generalize the geometry, treat multiple layers, and pursue a different solution technique for the second-order Fredholm integral equation. This equation takes the form of a Lippmann-Schwinger equation, and we apply a direct solution procedure using a discretization and a matrix inversion [16]. This method is exact, and only the discretization, enforced by the numerical implementation, introduces approximations. Furthermore, the method allows arbitrary index contrasts.

The paper is organized as follows. Section 2 presents our model and derivations, including the geometry to be analyzed, the modes and eigenmode expansions, the derivation of the integral equations and the solutions of these, reflection and transmission matrices, and the discretization of radiation modes. Section 3 presents the results of numerical simulations of the Purcell factor, Section 4 discusses our results and the perspectives, and Section $\underline{5}$ concludes the article.

\section{MODELING AND DERIVATIONS}

\section{A. Geometry}

We consider a micropillar as illustrated in the left panel of Fig. 1. The micropillar consists of a cavity and two distributed Bragg reflectors surrounded by vacuum. A dipole emitter is placed in the center of the cavity.

The structure is assumed uniform along $y$, implying a twodimensional (2D) problem in the $x z$ plane. The solution domain extends to infinity along both the positive and negative $x$ axis; i.e., there are no outer boundary conditions, and consequently no parasitic reflections. In the following sections, we shall establish the formalism for calculating the field due to the dipole emitter. The right panel in Fig. 1 shows an example of a calculated intensity profile, clearly illustrating a strong confinement of the field.

Throughout this paper we assume TE fields with the electric field polarized along the $y$ axis, although treatment of TM fields is not precluded by the method. This dictates the boundary conditions, and all calculations are described in terms of the electric field. Furthermore, uniformity along $y$ is assumed, which effectively reduces the solution of Maxwell's equations to variations in the $x z$ plane.

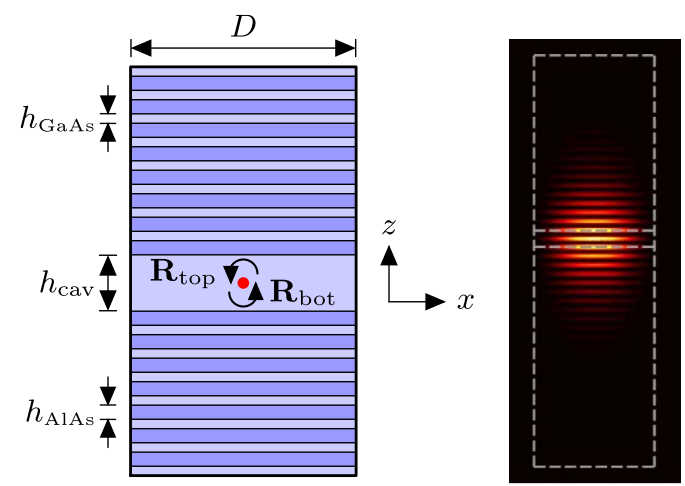

Fig. 1. (Color online) (Left) Emitter (red dot) inside a micropillar in the open geometry, illustrated in an $x z$ plane. Different colors represent different refractive indices. The micropillar is surrounded by vacuum. (Right) Calculated intensity profile of the micropillar design introduced in Section 3. Gray dashed lines outline the boundary of the micropillar.
While, for simplicity, we limit ourselves to a 2D geometry, the method can be generalized to three-dimensional (3D) geometries with analytical eigenmodes. We further discuss the applicability of the method in Section $\underline{4}$.

\section{B. Modeling of Purcell Factor}

As will be evident later in this section, the calculation of the Purcell factor requires an accurate simulation of the electric field, and, as discussed in Section 1, the calculation of the field using PML techniques is not guaranteed to converge to the correct open geometry field. Hence, the determination of the Purcell factor using our open geometry technique is an excellent means to assess the formalism.

We denote the SER of an emitter in an arbitrary optical environment as $\gamma$. The effect of the environment can be quantified by the relative enhancement or inhibition of $\gamma$, known as the Purcell factor, $F_{p}[\underline{2}]$ :

$$
F_{p}=\frac{\gamma}{\gamma_{0}}
$$

where $\gamma_{0}$ is the decay rate of the emitter when located in a homogeneous environment. The Purcell factor is closely related to the cavity quality factor, $Q$, and mode volume, $V$, through [17]

$$
F_{p}=\frac{Q}{V} \frac{3 \lambda^{3}}{4 \pi^{2} n^{3}},
$$

where $\lambda$ and $n$ are the wavelength in vacuum and the refractive index of the material in the cavity, respectively. It is well known that high values of $Q$ are desirable to obtain viable single-photon sources [12] and likewise that the computation of $Q$ is a demanding task. Having related $F_{p}$ to $Q$ above, it is apparent that accurate modeling of $F_{p}$ is needed.

Assuming a quantum emitter of ideal quantum efficiency, $F_{p}$ may equally well be determined as the enhancement or inhibition of the power radiated by an emitter, such that [18]

$$
F_{p}=\frac{P}{P_{0}},
$$

where $P$ and $P_{0}$ are the power radiated by the emitter in the cavity and in a homogeneous environment, respectively. The modeling of $F_{p}$ is then reduced to the determination of $P$ and $P_{0}$.

If we consider a dipole emitter with harmonic time dependence and current density $\mathbf{J}=J_{0} \delta\left(\mathbf{r}-\mathbf{r}_{0}\right) \hat{\mathbf{y}}$, the power radiated by this dipole may be determined as [18]

$$
P=-\frac{J_{0}}{2} \operatorname{Re}\left(E_{y}\left(\mathbf{r}_{0}\right)\right),
$$

where $E_{y}\left(\mathbf{r}_{0}\right)$ is the $y$ component of the electric field due to the dipole evaluated at the position of the dipole. By using the result in Eq. (4), it is a simple matter to obtain an analytical value for $P_{0}$, independent of the refractive index in the uniform medium.

It should be noted that the assumption of uniformity along $y$ implies that the dipole emitter in reality represents a quantum wire and not a quantum dot.

The procedure for determining the electric field due to an arbitrary current source is outlined in $[\underline{8}, \underline{19}]$. Using the EET, the determination of the field in structures with multiple layers is carried out using a scattering-matrix formalism 
[20], and in this context reflection and transmission matrices, $\mathbf{R}$ and $\mathbf{T}$, must be determined. This is the objective of the following sections.

\section{Modes and Eigenmode Expansion}

As discussed in Subsection 2.A, we aim to determine the field resulting from a dipole emitter inside a cavity in a micropillar; see Fig. 1. To that end, we initially consider the reduced problem of two arbitrary and adjacent layers in the micropillar. Two such layers are shown in Fig. 2. Notice that the cladding regions that were not shown explicitly in the preceding figure are now included. Although the cladding regions of the micropillar in Fig. 1 are vacuum in all layers, for generality we here allow for different cladding regions in the two layers, with refractive indices $n_{\mathrm{cl}}^{\{1\}}$ and $n_{\mathrm{cl}}^{\{2\}}$, respectively.

The $q$ th waveguide layer with refractive indices $n_{\mathrm{cl}}^{\{q\}}<n_{\mathrm{co}}^{\{q\}}$ in the cladding and core regions, respectively, supports a finite number, $N_{q}$, of guided modes, $U_{j}^{\{q\}}(x)$, and a continuum of radiation modes, $\psi_{m}^{\{q\}}(x, \rho)$. The radiation modes can be viewed as waves impinging from $x \rightarrow+\infty(x \rightarrow-\infty)$ for $m=$ $1(m=2)$. The modes are uniquely characterized by their $x$-propagation constants, $\left\{h_{j}^{\{q\}}, h_{j}^{\{q\}, \text { co }}\right\}$ and $\left\{\rho, \rho_{\text {co }}^{\{q\}}\right\}$ for guided modes and radiation modes, respectively. Superscript and subscript "co" on the $x$-propagation constants refer to the core region of the waveguide, while no superscript/subscript implicitly denotes cladding regions. These, the corresponding $z$-propagation constants, $\beta_{j}^{\{q\}}$ and $\beta^{\{q\}}(\rho)$, and formal definitions of the modes are presented in Appendix A.

We assume that illumination is caused by the excitation (1) of a guided mode, $U_{\hat{j}}^{\{1\}}(x)$, or (2) of a radiation mode, $\psi_{\hat{m}}^{\{1\}}(\hat{\rho}, x)$, that propagates in the positive $z$ direction in layer 1 ; see Fig. 2 . When illumination by a guided mode is considered, the electric fields in layers 1 and 2 are termed $E^{\{1\}}(x, z)$ and $E^{\{2\}}(x, z)$, respectively, and these take the following forms:

$$
\begin{aligned}
& E^{\{1\}}(x, z)=U_{\hat{j}}^{\{1\}}(x) e^{-i \beta_{j} z}+\sum_{j_{1}=1}^{N_{1}} R_{j_{1} \hat{j}}^{\{1\}} e^{i \beta_{j_{1}}^{\{1\}} z} U_{j_{1}}^{\{1\}}(x) \\
& +\sum_{m_{1}=1}^{2} \int_{0}^{\infty} R_{m_{1} \hat{j}}^{\{1\}}\left(\rho_{1}\right) e^{i \beta^{11\}}\left(\rho_{1}\right) z} \psi_{m_{1}}^{\{1\}}\left(x, \rho_{1}\right) \mathrm{d} \rho_{1}, \\
& E^{\{2\}}(x, z)=\sum_{j_{2}=1}^{N_{2}} T_{j_{2}}^{\{2\}} e^{-i \hat{j}_{2}\{2\}} U_{j_{2}}^{\{2\}}(x) \\
& +\sum_{m_{2}=1}^{2} \int_{0}^{\infty} T_{m_{2}, \hat{j}}^{\{2\}}\left(\rho_{2}\right) e^{-i \beta^{\{2\}}\left(\rho_{2}\right) z} \psi_{m_{2}}^{\{2\}}\left(x, \rho_{2}\right) \mathrm{d} \rho_{2} . \\
& z=\left.0 \longrightarrow \underset{n_{\mathrm{cl}}^{\{2\}}}{ }\right|_{-\frac{D}{2}}
\end{aligned}
$$

Fig. 2. (Color online) Two adjacent waveguide layers in the open geometry, illustrated in an $x z$ plane. The vertical arrow illustrates the illumination from layer 1 , while the dashed arrows indicate the scattering of the field at the interface.
We employ noncalligraphic letters for electric fields and reflection and transmission coefficients for illumination by a guided mode, while calligraphic letters are used when illumination is caused by a radiation mode. $E^{\{1\}}(x, z)$ and $E^{\{2\}}(x, z)$ depend implicitly on the guide mode illumination in$\operatorname{dex}, \hat{j}$, but we suppress this dependence for brevity. Equations (5a) and ( $\underline{5 b})$ coincide with [14] but are generalized to the case of two waveguide layers with arbitrary numbers of guided modes.

Illumination by a radiation mode has not previously been described in the literature. Since radiation modes cannot be excited individually in actual structures, but only as continuous superpositions, the simple interpretation applicable to illumination by guided modes cannot be transferred directly. However, it is conceptually identical to the wellknown study of illumination of an interface by plane waves; see for instance [21], in which the Fourier components of the field are considered separately. Likewise, the concept of Fresnel refraction at an interface is an example of illumination using a plane wave. The expansions are analogous to those for illumination by a guided mode, namely,

$$
\begin{aligned}
\mathcal{E}^{\{1\}}(x, z)= & \psi_{\hat{m}}^{\{1\}}(x, \hat{\rho}) e^{-i \beta^{\{1\}}(\hat{\rho}) z}+\sum_{j_{1}=1}^{N_{1}} \mathcal{R}_{j_{1}, \hat{m}}^{\{1\}}(\hat{\rho}) e^{i \beta_{j_{1}}^{\{1\}} z} U_{j_{1}}^{\{1\}}(x) \\
& +\sum_{m_{1}=1}^{2} \int_{0}^{\infty} \mathcal{R}_{m_{1}, \hat{m}}^{\{1\}}\left(\rho_{1}, \hat{\rho}\right) e^{i \beta^{\{1\}}\left(\rho_{1}\right) z} \psi_{m_{1}}^{\{1\}}\left(x, \rho_{1}\right) \mathrm{d} \rho_{1}, \\
\mathcal{E}^{\{2\}}(x, z)= & \sum_{j_{2}=1}^{N_{2}} \mathcal{T}_{j_{2}, \hat{m}}^{\{2\}}(\hat{\rho}) e^{-i \beta_{j_{2}}^{\{2\}} z} U_{j_{2}}^{\{2\}}(x) \\
& +\sum_{m_{2}=1}^{2} \int_{0}^{\infty} \mathcal{T}_{m_{2}, \hat{m}}^{\{2\}}\left(\rho_{2}, \hat{\rho}\right) e^{-i \beta^{\{2\}}\left(\rho_{2}\right) z} \psi_{m_{2}}^{\{2\}}\left(x, \rho_{2}\right) \mathrm{d} \rho_{2},
\end{aligned}
$$

where $\mathcal{E}^{\{1\}}(x, z)$ and $\mathcal{E}^{\{2\}}(x, z)$ are the electric fields in layers 1 and 2 , respectively, caused by illumination from a radiation mode. We again suppress the explicit dependence of the electric fields in the two layers on the illumination mode.

We stress that $E^{\{i\}}(x, z)$ and $\mathcal{E}^{\{i\}}(x, z), i=1,2$, in Eqs. (ㅁ) do not represent a separation of the guided and radiation modes but refer to the total field at the interface for illumination using either a guided or a radiation mode. The distinction between these two illumination conditions will be justified in the following.

The physical interpretation of the reflection and transmission coefficients is that, e.g., $\mathcal{R}_{j_{1}, \hat{m}}^{\{1\}}(\hat{\rho})$ and $\mathcal{T}_{m_{2}, \hat{m}}^{\{2\}}\left(\rho_{2}, \hat{\rho}\right)$ dictate the reflection and transmission of the incoming mode, $\psi_{\hat{m}}^{\{1\}}(\hat{\rho}, x)$, into the $j_{1}$ th guided mode in layer 1 and into the $\psi_{m_{2}}^{\{2\}}\left(x, \rho_{2}\right)$ radiation mode in layer 2 , respectively.

In Subsection 2.D, the aperture field, which is the electric field in the interface plane at $z=0$, is defined and an integral equation for this field is presented.

\section{Aperture Field}

By demanding that the electric field is continuous across the interface at $z=0$, the aperture fields for illumination by a guided mode, $\Phi_{\hat{j}}(x)$, and by a radiation mode, $\Theta_{\hat{m}}(x, \hat{\rho})$, 
may be defined as follows by using the eigenmode expansions in Eqs. (5):

$$
\begin{gathered}
\Phi_{\hat{j}}(x) \equiv E^{\{1\}}(x, 0)=E^{\{2\}}(x, 0), \\
\Theta_{\hat{m}}(x, \hat{\rho}) \equiv \mathcal{E}^{\{1\}}(x, 0)=\mathcal{E}^{\{2\}}(x, 0) .
\end{gathered}
$$

The reflection and transmission coefficients are functions of the aperture field. The dependence can be derived by application of the orthonormality relations [Eqs. (A3)] and the continuity condition described by Eqs. (6). The resulting expressions are given in Appendix B.

From the above comments, it is apparent that the determination of the aperture fields is central in the eigenmode expansion of the electric fields. Applying the second boundary condition, namely that the electric field is differentiable in the aperture plane, and using the definitions of the reflection and transmission coefficients and the orthonormality and the completeness of the modes within one layer, Eqs. (A3)-(A4) lead to the following second-order Fredholm integral equations in the aperture fields:

$$
\begin{gathered}
\Phi_{\hat{j}}(x)=\Phi_{0, \hat{j}}(x)+\Lambda\left\langle K_{2}^{*}(x) \mid \Phi_{\hat{j}}\right\rangle, \\
\Theta_{\hat{m}}(x, \hat{\rho})=\Theta_{0, \hat{m}}(x, \hat{\rho})+\Lambda\left\langle K_{2}^{*}(x) \mid \Theta_{\hat{m}}(\hat{\rho})\right\rangle,
\end{gathered}
$$

where the overlap integral, $\langle g \mid f\rangle$, is defined in Eq. (A2), $\Lambda$ is the integral equation eigenvalue, $\Phi_{0 \hat{j}}(x)$ and $\Theta_{0, \hat{m}}(x, \hat{\rho})$ are the zeroth-order aperture fields (essentially, scaled versions of the incident modes), and $K_{2}(x) \equiv K_{2}\left(x^{\prime}, x\right)$ is the integral equation kernel, all defined in Eqs. (B2) in Appendix B. A procedure for the derivations of these integral equations can be found in [22]. It is noted that the integration variable in the above integrals is $x^{\prime}$.

The integral equations Eqs. (7) are of the same form as the Lippmann-Schwinger equation. Approximate perturbative solutions were considered in [14] in the limit of weakly guiding waveguides. Solutions were computed using a truncated Liouville-Neumann series solution including up to two terms, and the complexity and extent of these approximate expressions increase rapidly as more terms are included, in practice limiting the approach to weak guides. To describe arbitrary index contrasts, we will pursue a different approach, where we solve the integral equation directly by discretizing the radiation modes, which in any case is required by the numerical implementation, and invert a matrix. This approach is presented in Subsection 2.E.

\section{E. Solution of Lippmann-Schwinger Equation}

In this section, the aperture fields are expanded on the complete set of eigenmodes from the illumination layer, layer 1 , and upon discretization of the radiation modes, the integral equations are converted into linear algebraic equations in the expansion coefficients. The solution of this system of equations involves the inversion of a matrix $\mathbf{K}$, to be introduced. The procedure follows steps similar to those in $[\underline{16}, \underline{23}]$.

The procedure is the same for Eqs. (7a) and (7b) and is outlined below for Eq. (7a). The aperture field and the zerothorder aperture field are expanded on the modes as follows:

$$
\begin{gathered}
\Phi_{\hat{j}}(x)=\sum_{j_{1}=1}^{N_{1}} c_{j_{1}} U_{j_{1}}^{\{1\}}(x)+\sum_{m_{1}=1}^{2} \int_{0}^{\infty} c_{m_{1}}(\rho) \psi_{m_{1}}^{\{1\}}(x, \rho) \mathrm{d} \rho, \\
\Phi_{0, j}(x)=\sum_{j_{1}=1}^{N_{1}} c_{j_{1}}^{0} U_{j_{1}}^{\{1\}}(x)+\sum_{m_{1}=1}^{2} \int_{0}^{\infty} c_{m_{1}}^{0}(\rho) \psi_{m_{1}}^{\{1\}}(x, \rho) \mathrm{d} \rho .
\end{gathered}
$$

For a specified guided illumination mode, $\Phi_{0, \hat{j}}(x)$ is known, whereby the coefficients $c_{j_{1}}^{0}$ and $c_{m_{1}}^{0}(\rho)$ are also known. The coefficients $c_{j_{1}}$ and $c_{m_{1}}(\rho)$, specifying the aperture field, remain to be determined. Insertion of these expansions into Eq. (7a) and application of the orthonormality relations from Eqs. (A3) yield the following equations in the unknown coefficients:

$$
\begin{aligned}
c_{j_{1}}= & c_{j_{1}}^{0}+\Lambda \int_{-\infty}^{\infty} \int_{-\infty}^{\infty} K_{2}\left(x^{\prime}, x\right) \\
& \times\left\{\sum_{j_{1}=1}^{N_{1}} c_{j_{1}} U_{j_{1}}^{\{1\}}\left(x^{\prime}\right)+\sum_{m_{1}=1}^{2} \int_{0}^{\infty} c_{m_{1}}(\rho) \psi_{m_{1}}^{\{1\}}\left(x^{\prime}, \rho\right) \mathrm{d} \rho\right\} \\
& \times\left(U_{j_{1}{ }^{\prime}}^{\{1\}}(x)\right)^{*} \mathrm{~d} x^{\prime} \mathrm{d} x \\
c_{m_{1}{ }^{\prime}}\left(\rho^{\prime}\right)= & c_{m_{1}{ }^{\prime}}^{0}\left(\rho^{\prime}\right)+\Lambda \int_{-\infty}^{\infty} \int_{-\infty}^{\infty} K_{2}\left(x^{\prime}, x\right) \\
& \times\left\{\sum_{j_{1}=1}^{N_{1}} c_{j_{1}} U_{j_{1}}^{\{1\}}\left(x^{\prime}\right)+\sum_{m_{1}=1}^{2} \int_{0}^{\infty} c_{m_{1}}(\rho) \psi_{m_{1}}^{\{1\}}\left(x^{\prime}, \rho\right) \mathrm{d} \rho\right\} \\
& \times\left(\psi_{m_{1}{ }^{\prime}}^{\{1\}}\left(x, \rho^{\prime}\right)\right)^{*} \mathrm{~d} x^{\prime} \mathrm{d} x .
\end{aligned}
$$

Once the radiation modes are discretized into $L$ modes, $\rho \in \mathbb{R}_{+} \rightarrow \rho \in\left\{\rho_{l}\right\}_{l=1}^{L}$, which in any case is required upon numerical implementation of the formalism, Eqs. (9a) and (9b) take the same form. The result can be summarized in the following compact equation:

$$
d_{k^{\prime}}=d_{k^{\prime}}^{0}+\sum_{k=1}^{N_{1}+2 L} K_{k^{\prime}, k} d_{k},
$$

where

$$
\begin{gathered}
d_{k}=\left\{\begin{array}{ll}
c_{k} & 1 \leq k \leq N_{1} \\
c_{1}\left(\rho_{k-N_{1}}\right) & N_{1}+1 \leq k \leq N_{1}+L \\
c_{2}\left(\rho_{k-N_{1}-L}\right) & N_{1}+L+1 \leq k \leq N_{1}+2 L
\end{array},\right. \\
d_{k}^{0}=\left\{\begin{array}{ll}
c_{k}^{0} & 1 \leq k \leq N_{1} \\
c_{1}^{0}\left(\rho_{k-N_{1}}\right) & N_{1}+1 \leq k \leq N_{1}+L \\
c_{2}^{0}\left(\rho_{k-N_{1}-L}\right) & N_{1}+L+1 \leq k \leq N_{1}+2 L
\end{array} .\right.
\end{gathered}
$$

The definitions of $K_{k^{\prime}, k}$ are given in Appendix $\underline{\mathrm{C}}$. By defining vectors with elements $d_{k^{\prime}}$ and $d_{k^{\prime}}^{0}$ and a matrix with elements $K_{k^{\prime}, k}$, the above equation can be formulated as a matrix equation in all of the unknown coefficients:

$$
\boldsymbol{d}=\boldsymbol{d}^{0}+\mathbf{K} \boldsymbol{d}
$$

from which

$$
\boldsymbol{d}=(\mathbf{I}-\mathbf{K})^{-1} \boldsymbol{d}^{0}
$$


As mentioned earlier, $\Phi_{0 \hat{j}}$, defined in Eq. (B2b), is merely a scaled version of the incoming mode, and to express $\boldsymbol{d}$ explicitly in terms of the expansion coefficients of the incoming mode, $d^{\text {in }}$, we have

$$
d^{0}=\zeta d^{\text {in }}
$$

where $\zeta$ is a diagonal matrix with elements

$$
\zeta_{k k}=-2 \Lambda \begin{cases}\beta_{k}^{\{1\}} & 1 \leq k \leq N_{1} \\ \beta_{1}\left(\rho_{k-N_{1}}\right) & N_{1}+1 \leq k \leq N_{1}+L \\ \beta_{1}\left(\rho_{k-N_{1}-L}\right) & N_{1}+L+1 \leq k \leq N_{1}+2 L\end{cases}
$$

With this notation Eq. (13) becomes

$$
\boldsymbol{d}=(\mathbf{I}-\mathbf{K})^{-1} \boldsymbol{\zeta} \boldsymbol{d}^{\mathrm{in}} .
$$

Having obtained the vector $\boldsymbol{d}$, the reflection and transmission matrices may finally be expressed; see Subsection 2.F.

\section{F. Reflection and Transmission Matrices}

The aperture field, described by the elements in $\boldsymbol{d}$, can be expressed as the sum of the incoming field, $\boldsymbol{d}^{\text {in }}$, and the reflected field, described at this point by an unknown coefficient vector, $\boldsymbol{d}^{r}$ :

$$
\boldsymbol{d}=\boldsymbol{d}^{\mathrm{in}}+\boldsymbol{d}^{r} .
$$

The coefficients for the reflected field are given via

$$
\boldsymbol{d}^{r}=\mathbf{R} \boldsymbol{d}^{\text {in }}
$$

where $\mathbf{R}$ is the reflection matrix. Combining Eqs. (16-18) gives the reflection matrix in terms of $\mathbf{K}$ :

$$
\mathbf{R}=(\mathbf{I}-\mathbf{K})^{-1} \boldsymbol{\zeta}-\mathbf{I}
$$

The calculation of the corresponding transmission matrix, $\mathbf{T}$, is straightforward. Since the aperture field in Eqs. (8) is expanded on modes from layer 1, a change of basis matrix is needed to express it in layer 2 . We thus define a matrix $\mathbf{M}$ that changes from the basis in layer 1 to the basis in layer 2 , that is, $\boldsymbol{d}^{\{2\}}=\mathbf{M} \boldsymbol{d}^{\{1\}}$, and the transmission matrix takes the form $\mathbf{T}=\mathbf{M}(\mathbf{I}-\mathbf{K})^{-1} \boldsymbol{\zeta}$.

When $\mathbf{R}$ and $\mathbf{T}$ have been determined, the electric field inside the cavity can be determined using the scattering-matrix formalism. Using the result in Eq. (4), this permits the determination of the radiated power and hence of the Purcell factor.

\section{G. Nonuniform Discretization}

The evaluation of Eq. (4) requires a summation over the discrete set of guided modes as well as a semi-infinite integral over the continuum of radiation modes. The evaluation of the semi-infinite $\rho$ integral necessarily requires a discretization, and represents the only approximation in the method.

A key advantage of our formalism is the freedom to choose the discretization in $\rho$, as opposed to the fixed set of modes that the artificial boundary conditions in a closed geometry impose. An intuitively efficient discretization is obtained by considering a uniform layer in which radiation in any direction in the $x z$ plane is equally likely. Thus, contributions to the total propagation constant $n k_{0}=\sqrt{\rho^{2}+\beta^{2}}$ from $\rho$ and $\beta$ should be weighed equally, and only propagating modes contribute to the emitted power, such that the semi-infinite integral is reduced to an integral over $0<\rho<n k_{0}$. To ensure an equal weighting of all directions, it is appropriate to choose an equidistant angular discretization:

$$
\rho=n k_{0} \cos (\theta)
$$

with $\theta$ given as a discrete set in the range $\theta \in[0, \pi / 2]$. We denote this discretization as the $\theta$ discretization. Figure (3) shows the equal angular spacing, and it is apparent that the $\rho$ spacing becomes increasingly dense as $\rho$ approaches $n k_{0}$.

Obviously, in more complicated multilayered waveguide structures, the above considerations do not hold strictly; for instance we expect all $\rho$ values to contribute and certain emission directions to be favored. However, since more complicated structures can essentially be thought of as perturbations of the uniform layer, we can expect to preserve many of the core features. Consequently, we pursue a discretization identical to that in Eq. (20) in the range $0<\rho<n_{\mathrm{cl}} k_{0}$. The remaining semi-infinite integration interval, $n_{\mathrm{cl}} k_{0}<\rho<\infty$, is cut off at $\rho_{\text {cut }}$, chosen according to convergence tests, and a reversed $\theta$ discretization, where the density of sampling points decreases as $\rho$ increases, is applied in this range.

\section{NUMERICAL RESULTS}

In this section, we present results for the Purcell factor in a micropillar geometry obtained using the open geometry formalism developed in Section 2. Results have been computed using an equidistant discretization of the radiation modes as well as the $\theta$ discretization outlined in Subsection 2.G, and $\mathbf{K}$ has been evaluated numerically. Comparison of the results from the two discretization methods demonstrates the strengths of the $\theta$ discretization and the benefits of free choice of discretization.

We consider a GaAs/AlAs micropillar geometry similar to the micropillar structures considered in [24], with a dipole emitter positioned symmetrically centered in a cavity layer of GaAs [25]. The refractive indices of the alternating layers of GaAs and AlAs are chosen as 3.495 and 2.94, respectively, according to a wavelength of $\lambda=0.95 \mu \mathrm{m}$. The micropillar is placed in vacuum, such that $n_{\mathrm{cl}}=1$ for all layers. The

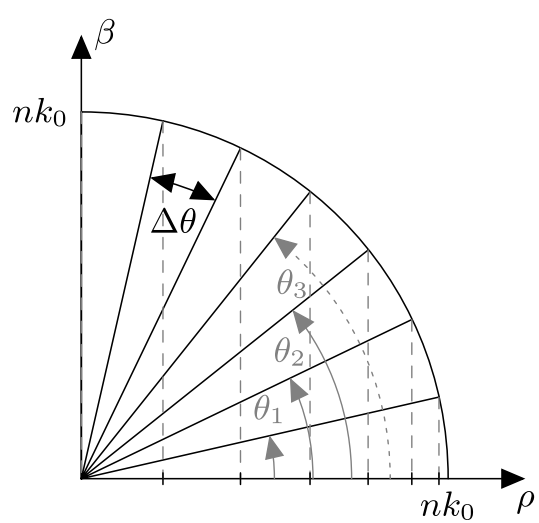

Fig. 3. Illustration of the relation $n k_{0}=\sqrt{\rho^{2}+\beta^{2}}$ in the $(\rho, \beta)$ plane, where the quarter circle represents the propagation constant $n k_{0}$. Solid lines indicate the equidistant angular spacing used in the $\theta$ discretization. 
structure is illustrated in Fig. 1 . The diameter of the micropillar is $D=2 \mu \mathrm{m}$. The total number of GaAs/AlAs distributed Bragg reflector (DBR) layer pairs are chosen as 25 and 20 for the bottom and top DBR mirrors, respectively. The thickness of each layer is determined according to the design in [24], requiring a quarter-wave optical thickness, $h_{i}=$ $\overline{\lambda /}\left(4 \bar{n}_{i}\right)$ for the $i$ th DBR layer, with $\bar{n}_{i}=\beta_{1}^{\{i\}} / k_{0}$ being the effective refractive index of the fundamental mode of the $i$ th layer. Similarly, the cavity thickness is chosen to equal an entire wavelength of the fundamental mode; that is, $h_{\text {cav }}=\lambda / \bar{n}_{\mathrm{GaAs}}$.

In each simulation, a cut-off value, $\rho_{\text {cut }}$, has been chosen based on convergence checks. If the distance from the dipole to the scattering surface, or from one scattering surface to another, is short, evanescent modes are more likely to scatter to propagating modes (and thus contribute to the emitted power), and we therefore expect that $\rho_{\text {cut }}$ should increase as the thinnest layer thickness $\min \left(h_{\text {cav }}, h_{i}\right)$ is decreased. In accordance with this and convergence checks, the cut-off value has been chosen as $\rho_{\text {cut }}=3.5 n_{\text {cl }} k_{0}$.

Results are computed as a function of the total number of included modes, i.e., the sum of the number of guided modes and the number of discretized radiation modes included in the simulation.

Results for the Purcell factor of the micropillar, computed by use of the matrix inversion procedure, are shown in Fig. 4 . Convergence is observed for both discretization methods. However, there is a distinct difference in the pattern of convergence. The $\theta$-discretization results display an initial transient oscillation but exhibits a uniform convergence after roughly 100 included modes. The equidistant discretization has the same initial transient behavior but continues to exhibit oscillations.

The origin of these oscillations can be explained by an analogy with the closed geometry. Upon discretization of the radiation modes with the equidistant method, the set of sampled propagation constants $\left\{\rho_{i}\right\}_{i}$ constitutes a set of numbers with a common divisor, $\Delta \rho$. Consequently, the set of eigenmodes, in this case of the form $\left\{\exp \left( \pm i \rho_{i} x\right)\right\}_{i}$, shares a common period $L_{\text {rep }}=2 \pi / \Delta \rho$. As a result, the far field calculated via the equidistant method will exhibit a period of repetition. In essence, the equidistant open geometry then emulates a periodic boundary domain. Accordingly, many of the same weaknesses are shared between the two other-

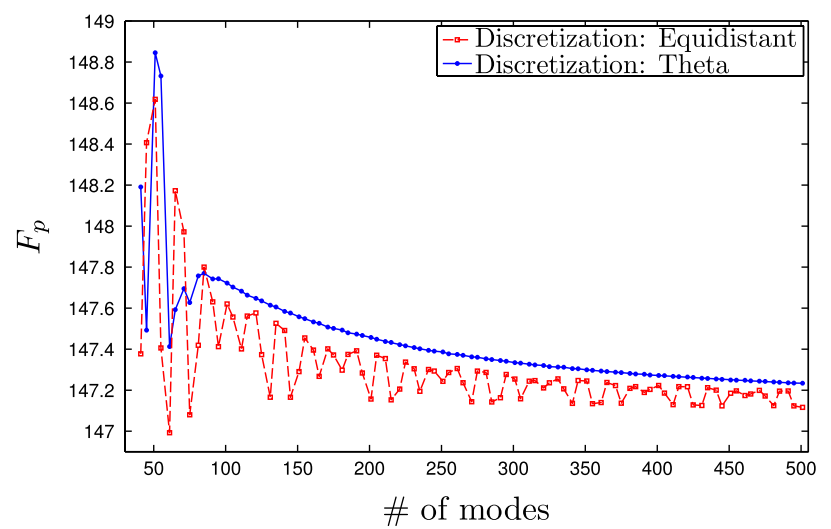

Fig. 4. (Color online) Simulation results for the Purcell factor. Results are presented as a function of the number of included modes for both discretization methods. wise distinct approaches-here exemplified by a strong and persistent oscillatory behavior of the $F_{p}$ simulations. Contrary to this, the far field calculated via the $\theta$ discretization exhibits no overall periodicity, since the set $\left\{\exp \left( \pm i \rho_{i} x\right)\right\}_{i}$ shares no common period. Furthermore, we obtain no persistent oscillation in $F_{p}$ versus the number of included modes.

The field intensity profile of the micropillar is depicted in the right panel of Fig. 1 , computed using the $\theta$-discretization method.

Simulations with 1000 included modes yield a Purcell factor, $F_{p}$, of 147.13 and 147.16 for equidistant and $\theta$ sampling, respectively. With the $\theta$ discretization (and possibly also with the equidistant sampling), our open geometry formalism presents a tool that, in principle, is capable of computing the Purcell factor to an arbitrary precision. A relative difference of approximately $1 \%$ is obtained with 40 included modes, demonstrating a high degree of accuracy and convergence even with few included modes.

In this work, we have performed $2 \mathrm{D}$ calculations, and we now compare our characteristic parameters with those obtained using full 3D simulations. First, using Eqs. (1) and (2) in [12], we compute the $Q$ factor for the cavity and find $Q=34500$. Using $F_{p}=147.16$ and solving Eq. (2) for the mode volume, $V$, we then determine a numerical value for the mode volume that the micropillar would have in the corresponding $3 \mathrm{D}$ geometry:

$$
V=\frac{Q}{F_{p}} \frac{3 \lambda^{3}}{4 \pi^{2} n^{3}}=0.36 \mu \mathrm{m}^{3} .
$$

This number is in good agreement with the mode volume computed using a full 3D model [26].

\section{DISCUSSION}

The formalism established in this paper holds both physical and computational advantages as compared to other established simulation techniques such as FDTD and FEM. First, the electric fields are expanded on analytical eigenmodes, which ensures more accurate and physically intuitive fields. Also, the search for roots of a transcendental equation is only necessary for the guided modes. The cumbersome search routines [8] necessary to determine propagation constants of radiation modes in PML formulations are avoided. Furthermore, it is a major advantage of the proposed method that it is formulated for an open geometry, which is the natural setting for optical cavities given the propagation characteristics of photons.

The largest drawback of the method in our current implementation is the need to evaluate the semi-infinite and illbehaved $\rho$ integrals occurring in Eqs. (C2-C3) numerically. This represents, by far, the bulk of the computation of the $\mathbf{K}$ matrix and by extension the bulk of any open geometry matrix inversion computation. Any analytical or approximate solution of these integrals would potentially elevate the method from a rapidly converging and accurate scheme to a computationally efficient scheme as well. A different approach for reducing the computational requirements exists via assessment of the error of the field computation via insertion of the computed field into Eqs. (ㅁ). Accurate assessment of the error could potentially allow for down-adjustment, e.g., of the required accuracy in the numerical evaluation of $\rho$ integrals. 
We stress that our formalism applies to any geometry with analytical eigenmodes. $2 \mathrm{D}$ results have been presented for simplicity, but the method can readily be extended to 3D structures for which analytical eigenmodes are available. This is the case for 3D structures with rotational symmetry that essentially reduce to $2 \mathrm{D}$ problems due to angular momentum decoupling, and for this reason we expect no performance penalty for these structures. Likewise, for 3D structures with elliptical cross section analytical eigenmodes exist [27], and our method thus applies to this geometry as well.

\section{CONCLUSION}

We have introduced a formalism to compute the electric field in arbitrary step-index profiles using an open geometry. In the open geometry, the eigenmodes are constituted by a finite number of guided modes and a continuum of radiation modes, and the electric field is expanded on these analytical modes, ensuring high accuracy. The electric field in multilayered structures is computed using the standard $S$-matrix formalism by employing reflection and transmission matrices. Determination of these matrices involves the solution of a Lippmann-Schwinger equation. The solution procedure requires a discretization of the radiation modes. A central advantage of our formalism, as compared to formalisms with closed or periodic boundary conditions, is the freedom to choose this discretization. As an example of the possibilities of the formalism, we model and calculate the Purcell factor, $F_{p}$, due to a quantum wire embedded in a GaAs/AlAs micropillar. We calculate $F_{p}$ as a function of the number of included modes using two different discretization techniques. When employing the $\theta$ discretization, the results display uniform convergence, while an oscillatory convergence is observed when applying an equidistant discretization. These results pave the way for accurate modeling of open geometries in 2D and 3D structures where analytical eigenmodes are available.

\section{APPENDIX A: EIGENMODES}

\section{Definitions of Modes}

The modes in the $q$ th waveguide layer, presented in Subsection 2.C, take the following forms: where $N_{j}^{\{q\}}$ and $\mathcal{N}_{m}^{\{q\}}(\rho)$ are real normalization constants, $\left(h_{j}^{\{q\}, \mathrm{cl} / \mathrm{co}}\right)^{2}=\left(n_{\mathrm{cl} / \mathrm{co}}^{\{q\}} k_{0}\right)^{2}-\left(\beta_{j}^{\{q\}}\right)^{2}$ are the $x$-propagation constants of the $j$ th guided mode in the cladding and core regions, respectively, and $0<\rho<\infty$ and $\left(\rho_{\mathrm{co}}^{\{q\}}\right)^{2}=k_{0}^{2}\left[\left(n_{\mathrm{co}}^{\{q\}}\right)^{2}-\right.$ $\left.\left(n_{\mathrm{cl}}^{\{q\}}\right)^{2}\right]+\rho^{2}$ are the $x$-propagation constants of a radiation mode in the cladding and core regions, respectively. The $z$ propagation constants of the guided and radiation modes are $\beta_{j}^{\{q\}} \in \mathbb{R}_{+}$and $\beta^{\{q\}}(\rho)^{2}=\left(n_{\mathrm{cl}}^{\{q\}} k_{0}\right)^{2}-\rho^{2}$, respectively. The former is determined numerically from the $x$-boundary conditions of the guided modes, while the latter may be either positive real $\left(0<\beta^{\{q\}}(\rho)<n_{\mathrm{cl}}^{\{q\}} k_{0}\right)$ or negative imaginary, which corresponds to propagating or exponentially decaying radiation modes, respectively. Finally, the radiation modes may be interpreted as illumination from the left $[m=$ $\left.1: a_{1}^{\{q\}, 1}(\rho) \neq 0, b_{1}^{\{q\}, 3}(\rho)=0\right]$ or from the right $\left[m=2: a_{2}^{\{q\}, 1}\right.$ $\left.(\rho)=0, b_{2}^{\{q\}, 3}(\rho) \neq 0\right]$. The orthonormality and completeness of the modes within one layer, used in Subsection 2.D, are presented in Appendix $\underline{B}$.

\section{Mode Orthogonality and Completeness}

Inner products, used to express overlap integrals, are denoted by the usual bracket notation:

$$
\langle g \mid f\rangle=(\langle f \mid g\rangle)^{*} \equiv \int_{-\infty}^{\infty} f(x) g^{*}(x) \mathrm{d} x
$$

With this notation, the orthonormality of the modes introduced in Subsection 2.C can be expressed as [14]

$$
\left\langle U_{k}^{\{q\}} \mid U_{j}^{\{q\}}\right\rangle=\delta_{j k}
$$

$$
\left\langle\psi_{m}^{\{q\}}(\rho) \mid U_{j}^{\{q\}}\right\rangle=0
$$

$$
\left\langle\psi_{m^{\prime}}^{\{q\}}\left(\rho^{\prime}\right) \mid \psi_{m}^{\{q\}}(\rho)\right\rangle=\delta_{m m^{\prime}} \delta\left(\rho-\rho^{\prime}\right),
$$

while the completeness of the set of modes becomes

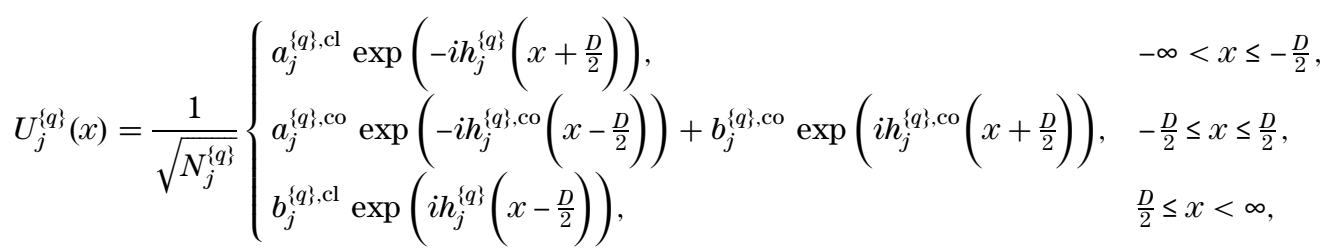

$$
\psi_{m}^{\{q\}}(x, \rho)=\frac{1}{\sqrt{\mathcal{N}_{m}^{\{q\}}(\rho)}} \begin{cases}a_{m}^{\{q\}, 1}(\rho) \exp (-i \rho x)+b_{m}^{\{q\}, 1}(\rho) \exp (i \rho x), & -\infty<x \leq-\frac{D}{2}, \\ a_{m}^{\{q\}, 2}(\rho) \exp \left(-i \rho_{\mathrm{co}}^{\{q\}} x\right)+b_{m}^{\{q\}, 2}(\rho) \exp \left(i \rho_{\mathrm{co}}^{\{q\}} x\right), & -\frac{D}{2} \leq x \leq \frac{D}{2}, \\ a_{m}^{\{q\}, 3}(\rho) \exp (-i \rho x)+b_{m}^{\{q\}, 3}(\rho) \exp (i \rho x), & \frac{D}{2} \leq x<\infty,\end{cases}
$$




$$
\begin{aligned}
\delta\left(x-x^{\prime}\right)= & \sum_{j=1}^{N_{q}} U_{j}(x)\left(U_{j}^{\{q\}}\left(x^{\prime}\right)\right)^{*} \\
& +\sum_{m=1}^{2} \int_{0}^{\infty} \psi_{m}^{\{q\}}(x, \rho)\left(\psi_{m}^{\{q\}}\left(x^{\prime}, \rho\right)\right)^{*} \mathrm{~d} \rho .
\end{aligned}
$$

\section{APPENDIX B: APERTURE FIELD INTEGRAL EQUATION}

\section{Reflection and Transmission Coefficients}

By requiring continuity of the aperture fields in Eqs. (6) and applying the orthonormality of the modes, the following definitions of the reflection and transmission coefficients are obtained:

$$
\begin{gathered}
R_{j_{1} \hat{j}}^{\{1\}}=\left\langle U_{j_{1}}^{\{1\}} \mid \Phi\right\rangle-\delta_{j_{1} \hat{j}}, \\
\mathcal{R}_{j_{1}, \hat{m}}^{\{1\}}(\hat{\rho})=\left\langle U_{j_{1}}^{\{1\}} \mid \Theta_{\hat{m}}(\hat{\rho})\right\rangle, \\
R_{m_{1}, \hat{j}}^{\{1\}}\left(\rho_{1}\right)=\left\langle\psi_{m_{1}}^{\{1\}}\left(\rho_{1}\right) \mid \Phi\right\rangle, \\
\mathcal{R}_{m_{1}, \hat{m}}^{\{1\}}\left(\rho_{1}, \hat{\rho}\right)=\left\langle\psi_{m_{1}}^{\{1\}}\left(x, \rho_{1}\right) \mid \Theta_{\hat{m}}(\hat{\rho})\right\rangle-\delta_{m_{1} \hat{m}} \delta\left(\rho_{1}-\hat{\rho}\right), \\
T_{j_{2}, \hat{j}}^{\{2\}}=\left\langle U_{j_{2}}^{\{2\}} \mid \Phi\right\rangle, \\
\mathcal{T}_{j_{2}, \hat{m}}^{\{2\}}(\hat{\rho})=\left\langle U_{j_{2}}^{\{2\}} \mid \Theta_{\hat{m}}(\hat{\rho})\right\rangle, \\
T_{m_{2}, \hat{j}}^{\{2\}}\left(\rho_{2}\right)=\left\langle\psi_{m_{2}}^{\{2\}}\left(\rho_{2}\right) \mid \Phi\right\rangle, \\
\mathcal{R}_{j_{1}, \hat{m}}^{\{1\}}(\hat{\rho})=\left\langle\psi_{m_{2}}^{\{2\}}\left(\rho_{2}\right) \mid \Theta_{\hat{m}}(\hat{\rho})\right\rangle .
\end{gathered}
$$

\section{Definitions for Second-Order Fredholm Equation}

Definitions required in the integral equations for the aperture fields [Eqs. (7)] are given below:

$$
\begin{gathered}
\Lambda=-\frac{1}{k_{0}\left(n_{\mathrm{cl}}^{\{1\}}+n_{\mathrm{cl}}^{\{2\}}\right)}, \\
\Phi_{0, \hat{j}}(x)=\frac{2 \beta_{\hat{j}}^{\{1\}}}{k_{0}\left(n_{\mathrm{cl}}^{\{1\}}+n_{\mathrm{cl}}^{\{2\}}\right)} U_{\hat{j}}^{\{1\}}(x), \\
\Theta_{0, \hat{m}}(x, \hat{\rho})=\frac{2 \beta_{1}(\hat{\rho})}{k_{0}\left(n_{\mathrm{cl}}^{\{1\}}+n_{\mathrm{cl}}^{\{2\}}\right)} \psi_{\hat{m}}^{\{1\}}(x, \hat{\rho}),
\end{gathered}
$$

$$
\begin{aligned}
K_{2}\left(x^{\prime}, x\right)= & \sum_{j_{1}=1}^{N_{1}}\left(\beta_{j_{1}}^{\{1\}}-k_{0} n_{\mathrm{cl}}^{\{1\}}\right) U_{j_{1}}^{\{1\}}(x)\left(U_{j_{1}}^{\{1\}}\left(x^{\prime}\right)\right)^{*} \\
& +\sum_{m_{1}=1}^{2} \int_{0}^{\infty}\left(\beta_{1}\left(\rho_{1}\right)-k_{0} n_{\mathrm{cl}}^{\{1\}}\right) \psi_{m_{1}}^{\{1\}}\left(x, \rho_{1}\right) \\
& \times\left(\psi_{m_{1}}^{\{1\}}\left(x^{\prime}, \rho_{1}\right)\right)^{*} \mathrm{~d} \rho_{1} \\
& +\sum_{j_{2}=1}^{N_{2}}\left(\beta_{j_{2}}^{\{2\}}-k_{0} n_{\mathrm{cl}}^{\{2\}}\right) U_{j_{2}}^{\{2\}}(x)\left(U_{j_{2}}^{\{2\}}\left(x^{\prime}\right)\right)^{*} \\
& +\sum_{m_{2}=1}^{2} \int_{0}^{\infty}\left(\beta_{2}\left(\rho_{2}\right)-k_{0} n_{\mathrm{cl}}^{\{2\}}\right) \psi_{m_{2}}^{\{2\}}\left(x, \rho_{2}\right) \\
& \times\left(\psi_{m_{2}}^{\{2\}}\left(x^{\prime}, \rho_{2}\right)\right)^{*} \mathrm{~d} \rho_{2} .
\end{aligned}
$$

\section{APPENDIX C: MATRIX INVERSION}

\section{Elements of $\mathrm{K}$}

The matrix $\mathbf{K}$ was introduced in Subsection 2.E. It can be decomposed into four block matrices:

$$
\mathbf{K}=\left[\begin{array}{ll}
\mathbf{K}^{\mathrm{GG}} & \mathbf{K}^{\mathrm{RG}} \\
\mathbf{K}^{\mathrm{GR}} & \mathbf{K}^{\mathrm{RR}}
\end{array}\right],
$$

where the indices of the elements of the constituent block matrices vary according to

$$
\left[\begin{array}{cc}
K_{k^{\prime}, k}^{\mathrm{GG},} & K_{k^{\prime}, k}^{\mathrm{RG},}, \\
1 \leq k^{\prime} \leq N_{1}, & 1 \leq k^{\prime} \leq N_{1}, \\
1 \leq k \leq N_{1}, & N_{1}+1 \leq k \leq N_{1}+2 L, \\
K_{k^{\prime}, k}^{\mathrm{GR}}, & K_{k^{\prime}, k}^{\mathrm{RR}}, \\
N_{1}+1 \leq k^{\prime} \leq N_{1}+2 L, & N_{1}+1 \leq k^{\prime} \leq N_{1}+2 L, \\
1 \leq k \leq N_{1}, & N_{1}+1 \leq k \leq N_{1}+2 L,
\end{array}\right]
$$

The superscript notation is to be interpreted as follows. The first index indicates excitation mode type, and the second index indicates a contribution to the aperture field. For instance, $\mathbf{K}^{\mathrm{GR}}$ governs contributions to the aperture field from the radiation modes, due to excitation by a guided mode. The elements of each of the block matrices are expressed below:

$$
\begin{aligned}
K_{k^{\prime}, k}^{\mathrm{GG}}= & \Lambda\left[\left(\beta_{k}^{\{1\}}-k_{0} n_{\mathrm{cl}}^{\{1\}}\right) \delta_{k k^{\prime}}+\sum_{j_{2}=1}^{N_{2}}\left(\beta_{j_{2}}^{\{2\}}-k_{0} n_{\mathrm{cl}}^{\{2\}}\right)\right. \\
& \times\left\langle U_{j_{2}}^{\{2\}} \mid U_{k}^{\{1\}}\right\rangle\left\langle U_{k^{\prime}}^{\{1\}} \mid U_{j_{2}}^{\{2\}}\right\rangle \\
& +\sum_{m_{2}=1}^{2} \int_{0}^{\infty}\left(\beta_{2}\left(\rho_{2}\right)-k_{0} n_{\mathrm{cl}}^{\{2\}}\right)\left\langle\psi_{m_{2}}^{\{2\}}\left(\rho_{2}\right) \mid U_{k}^{\{1\}}\right\rangle \\
& \left.\times\left\langle U_{k^{\prime}}^{\{1\}} \mid \psi_{m_{2}}^{\{2\}}\left(\rho_{2}\right)\right\rangle \mathrm{d} \rho_{2}\right],
\end{aligned}
$$

$$
\begin{aligned}
K_{k^{\prime}, \tilde{k}}^{\mathrm{RG}}= & \Lambda \Delta \rho_{k-N_{1}-L_{k}}\left[\sum_{j_{2}=1}^{N_{2}}\left(\beta_{j_{2}}^{\{2\}}-k_{0} n_{\mathrm{cl}}^{\{2\}}\right)\right. \\
& \times\left\langle U_{j_{2}}^{\{2\}} \mid \psi_{\eta_{k}}^{\{1\}}\left(\rho_{k-N_{1}-L_{k}}\right)\right\rangle\left\langle U_{k^{\prime}}^{\{1\}} \mid U_{j_{2}}^{\{2\}}\right\rangle \\
& +\sum_{m_{2}=1}^{2} \int_{0}^{\infty}\left(\beta_{2}\left(\rho_{2}\right)-k_{0} n_{\mathrm{cl}}^{\{2\}}\right)\left\langle\psi_{m_{2}}^{\{2\}}\left(\rho_{2}\right) \mid \psi_{\eta_{k}}^{\{1\}}\left(\rho_{k-N_{1}-L_{k}}\right)\right\rangle \\
& \left.\times\left\langle U_{k^{\prime}}^{\{1\}} \mid \psi_{m_{2}}^{\{2\}}\left(\rho_{2}\right)\right\rangle \mathrm{d} \rho_{2}\right],
\end{aligned}
$$




$$
\begin{aligned}
K_{\tilde{k}^{\prime}, k}^{\mathrm{GR}}= & \Lambda\left[\sum_{j_{2}=1}^{N_{2}}\left(\beta_{j_{2}}^{\{2\}}-k_{0} n_{\mathrm{cl}}^{\{2\}}\right)\left\langle U_{j_{2}}^{\{2\}} \mid U_{k}^{\{1\}}\right\rangle\left\langle\psi_{\eta_{k^{\prime}}}^{\{1\}}\left(\rho_{k^{\prime}-N_{1}-L_{k^{\prime}}}\right) \mid U_{j_{2}}^{\{2\}}\right\rangle\right. \\
& +\sum_{m_{2}=1}^{2} \int_{0}^{\infty}\left(\beta_{2}\left(\rho_{2}\right)-k_{0} n_{\mathrm{cl}}^{\{2\}}\right)\left\langle\psi_{\eta_{k^{\prime}}}^{\{1\}}\left(\rho_{k^{\prime}-N_{1}-L_{k^{\prime}}}\right) \mid \psi_{m_{2}}^{\{2\}}\left(\rho_{2}\right)\right\rangle \\
& \left.\times\left\langle\psi_{m_{2}}^{\{2\}}\left(\rho_{2}\right) \mid U_{k}^{\{1\}}\right\rangle \mathrm{d} \rho_{2}\right], \\
K_{\tilde{k}^{\prime}, \tilde{k}}^{\mathrm{RR}}= & \Lambda\left[K_{\eta_{k^{\prime}}, \eta_{k}}^{\mathrm{ND}}\left(\rho_{k^{\prime}-N_{1}-L_{k^{\prime}}} \rho_{k-N_{1}-L_{k}}\right) \Delta \rho_{k-N_{1}-L_{k}}\right. \\
& \left.+K_{\eta_{k^{\prime}}, \eta_{k}}^{\mathrm{D}}\left(\rho_{k^{\prime}-N_{1}-L_{k^{\prime}}}\right) \delta_{k^{\prime}-L_{k^{\prime}}, k-L_{k}}\right]
\end{aligned}
$$

with

$$
\begin{aligned}
K_{m_{1}{ }^{\prime}, m_{1}}^{\mathrm{ND}}\left(\rho^{\prime}, \rho\right)= & \sum_{j_{2}=1}^{N_{2}}\left[\beta_{j_{2}}^{\{2\}}-k_{0} n_{\mathrm{cl}}^{\{2\}}\right]\left\langle\psi_{m_{1}{ }^{\prime}}^{\{1\}}\left(\rho^{\prime}\right) \mid U_{j_{2}}^{\{2\}}\right\rangle\left\langle U_{j_{2}}^{\{2\}} \mid \psi_{m_{1}}^{\{1\}}(\rho)\right\rangle \\
+ & \sum_{m_{2}=1}^{2}\left\{\int_{0}^{\infty}\left[\beta_{2}\left(\rho_{2}\right)-k_{0} n_{\mathrm{cl}}^{\{2\}}\right]\right. \\
& \times H_{m_{2}, m_{1}}^{\{2,1\}}\left(\rho_{2}, \rho\right) H_{m_{1}{ }^{\prime}, m_{2}}^{\{1,2\}}\left(\rho^{\prime}, \rho_{2}\right) \mathrm{d} \rho_{2} \\
+ & {\left[\beta_{2}(\rho)-k_{0} n_{\mathrm{cl}}^{\{2\}}\right] G_{m_{2}, m_{1}}^{\{2,1\}}(\rho) H_{m_{1}{ }^{\prime}, m_{2}}^{\{1,2\}}\left(\rho^{\prime}, \rho\right) } \\
+ & {\left.\left[\beta_{2}\left(\rho^{\prime}\right)-k_{0} n_{\mathrm{cl}}^{\{2\}}\right] H_{m_{2}, m_{1}}^{\{2,1\}}\left(\rho^{\prime}, \rho\right) G_{m_{1}{ }^{\prime}, m_{2}}^{\{1,2\}}\left(\rho^{\prime}\right)\right\},(\mathrm{C} 3 \mathrm{a}) } \\
K_{m_{1}{ }^{\prime}, m_{1}}^{\mathrm{D}}\left(\rho^{\prime}\right)= & \delta_{m_{1^{\prime}, m_{1}}}\left[\beta_{1}\left(\rho^{\prime}\right)-k_{0} n_{\mathrm{cl}}^{\{1\}}\right] \\
& +\sum_{m_{2}=1}^{2} G_{m_{2}, m_{1}}^{\{2,1\}}\left(\rho^{\prime}\right) G_{m_{1}{ }^{\prime}, m_{2}}^{\{1,2\}}\left(\rho^{\prime}\right)\left[\beta_{2}\left(\rho^{\prime}\right)-k_{0} n_{\mathrm{cl}}^{\{2\}}\right]
\end{aligned}
$$

To express the above elements, the following definitions have been applied:

$$
\begin{gathered}
\eta_{k}=\left\{\begin{array}{cc}
1 & N_{1}<k \leq N_{1}+L \\
2 & N_{1}+L<k \leq N_{1}+2 L
\end{array},\right. \\
L_{k}=\left\{\begin{array}{cc}
0 & N_{1}<k \leq N_{1}+L \\
L & N_{1}+L<k \leq N_{1}+2 L
\end{array},\right. \\
\tilde{k}=k-N_{1}-L_{k}, \\
\left\langle\psi_{m}^{\{q\}}(\rho) \mid \psi_{m^{\prime}}^{\left\{q^{\prime}\right\}}\left(\rho^{\prime}\right)\right\rangle=\delta\left(\rho-\rho^{\prime}\right) G_{m, m^{\prime}}^{\left\{q, q^{\prime}\right\}}(\rho)+H_{m, m^{\prime}}^{\left\{q, q^{\prime}\right\}}\left(\rho, \rho^{\prime}\right) .
\end{gathered}
$$

\section{ACKNOWLEDGMENTS}

J. Rosenkrantz de Lasson and T. Christensen thank Torben Roland Nielsen for valuable feedback and interest in our work. N. Gregersen acknowledges fruitful discussions with A. B. Manenkov. Support from the Villum Foundation via the Villum Kann Rasmussen Centre of Excellence NAnophotonics for TErabit Communications is gratefully acknowledged.

$\dagger$ These authors have contributed equally to this work.

\section{REFERENCES}

1. K. Vahala, “Optical microcavities," Nature 424, 839-846 (2003).

2. E. Purcell, "Spontaneous emission probabilities at radio frequencies,” Phys. Rev. 69, 681 (1946).

3. J. Claudon, J. Bleuse, N. S. Malik, M. Bazin, P. Jaffrennou, N. Gregersen, C. Sauvan, P. Lalanne, and J. M. Gérard, "A highly efficient single-photon source based on a quantum dot in a photonic nanowire," Nat. Photonics 4, 174-177 (2010).

4. A. Taflove and S. Hagness, Computational Electromagnetics: The Finite-Difference Time-Domain Method (Artech House, 2005).

5. J. Reddy, An Introduction to the Finite Element Method (McGraw-Hill Science/Engineering/Math, 2005).

6. E. Silberstein, P. Lalanne, J. P. Hugonin, and Q. Cao, "Use of grating theories in integrated optics,” J. Opt. Soc. Am. A 18, 2865-2875 (2001).

7. P. Bienstman and R. Baets, "Optical modelling of photonic crystals and VCSELs using eigenmode expansion and perfectly matched layers," Opt. Quantum Electron. 33, 327-341 (2001).

8. P. Bienstman, "Rigorous and efficient modelling of wavelength scale photonic components," Ph.D. thesis (University of Gent, Department of Information Technology, 2001).

9. J. P. Berenger, "A perfectly matched layer for the absorption of electromagnetic waves,” J. Comput. Phys. 114, 185-200 (1994)

10. N. Gregersen and J. Mørk, "An improved perfectly matched layer for the eigenmode expansion technique," Opt. Quantum Electron. 40, 957-966 (2008).

11. J. P. Hugonin and P. Lalanne, "Perfectly matched layers as nonlinear coordinate transforms: a generalized formalization," J. Opt. Soc. Am. A 22, 1844-1849 (2005).

12. N. Gregersen, S. Reitzenstein, C. Kistner, M. Strauss, C. Schneider, S. Höfling, L. Worschech, A. Forchel, T. R. Nielsen, J. Mørk, and J. M. Gérard, "Numerical and experimental study of the $Q$ factor of high- $Q$ micropillar cavities," IEEE J. Quantum Electron. 46, 1470-1483 (2010).

13. N. Bonod, E. Popov, and M. Neviére, "Differential theory of diffraction by finite cylindrical objects," J. Opt. Soc. Am. A 22, 481-491 (2005).

14. I. Tigelis and A. Manenkov, "Scattering from an abruptly terminated asymmetrical slab waveguide," J. Opt. Soc. Am. A 16, 523-532 (1999).

15. I. Tigelis and A. Manenkov, "Analysis of mode scattering from an abruptly ended dielectric slab waveguide by an accelerated iteration technique," J. Opt. Soc. Am. A 17, 2249-2259 (2000).

16. P. Kristensen, P. Lodahl, and J. Mørk, "Light propagation in finite-sized photonic crystals: multiple scattering using an electric field integral equation," J. Opt. Soc. Am. B 27, 228-237 (2010).

17. J. M. Gérard, "Solid-state cavity-quantum electrodynamics with self-assembled quantum dots," in Single Quantum Dots: Physics and Applications, P. Michler, ed. (Springer-Verlag, 2003), pp. 269-315.

18. L. Novotny and B. Hecht, Principles of Nano-Optics, 1st ed. (Cambridge University, 2006), Chap. 8, pp. 250-303.

19. A. Snyder and J. Love, Optical Waveguide Theory, 1st ed. (Chapman and Hall, 1983), Chap. 31, pp. 601-622.

20. L. Li, "Formulation and comparison of two recursive matrix algorithms for modeling layered diffraction gratings," J. Opt. Soc. Am. A 13, 1024-1035 (1996).

21. M. G. Moharam, E. B. Grann, D. A. Pommet, and T. K. Gaylord, "Formulation for stable and efficient implementation of the rigorous coupled-wave analysis of binary gratings," J. Opt. Soc. Am. A 12, 1068-1076 (1995).

22. N. K. Uzunoglu, C. N. Capsalis, and I. Tigelis, "Scattering from and abruptly terminated single-mode-fiber waveguide," J. Opt. Soc. Am. A 4, 2150-2157 (1987).

23. P. Kristensen, "Light-matter interaction in nanostructured materials," Ph.D. thesis (Technical University of Denmark, Department of Photonics Engineering, 2009).

24. P. Lalanne, J. P. Hugonin, and J. M. Gérard, "Electromagnetic study of the quality factor of pillar microcavities in the small diameter limit," Appl. Phys. Lett. 84, 4726-4728 (2004). 
25. S. Reitzenstein and A. Forchel, "Quantum dot micropillars," J. Phys. D 43, 033001 (2010).

26. M. Lermer, N. Gregersen, F. Dunzer, S. Reitzenstein, S. Höfling, J. Mørk, L. Worschech, M. Kamp, and A. Forchel, "Bloch-wave engineering of quantum dot micropillars for cavity quantum electrodynamics experiments," Phys. Rev. Lett. 108, 057402 (2012).

27. L. W. Li, H. G. Wee, and M. S. Leong, "Dyadic Green's functions inside/outside a dielectric elliptical cylinder: theory and application," IEEE Trans. Antennas Propag. 51, 564-574 (2003). 